\title{
Reacción a Moniliophthora roreri en Theobroma spp. en Caquetá, Colombia
}

\author{
Armando Sterling Cuéllar ${ }^{1}$, Marfi Andrea Hermida Daza ${ }^{1,2}$, Carlos Hernando Rodríguez León ${ }^{1}$, Yasodhara Marieth \\ Salas Tobón ${ }^{1}$, María Natali Nieto Guzmán ${ }^{1,2}$ Diego Ferney Caicedo Rodríguez ${ }^{1}$
}

${ }^{1}$ Instituto Amazónico de Investigaciones Científicas SINCHI Sede Florencia. Calle 17 No. 11-67, 2 Piso, Florencia, Caquetá (Colombia). ${ }^{2}$ Universidad de la Amazonia, Grupo de Micología GINMUA. Sede Principal. Avd. Circunvalación, Barrio El Porvenir. Florencia, Caquetá (Colombia).

Autor para correspondencia: Armando Sterling Cuéllar (asterling@sinchi.org.co)

Data de chegada: 21/08/2014. Aceito para publicação em: 29/06/2015.

$10.1590 / 0100-5405 / 2026$

\section{RESUMEN}

Sterling, C.A.; Hermida-Daza, M.A.; Rodriguez-León, C.H.; Salas-Tobón, Y. M.; Nieto-Guzmán, M.N.; Caicedo-Rodríguez, D.F. Reacción a Moniliophthora roreri en Theobroma spp. en Caquetá, Colombia. Summa Phytopathologica, v.41, n.3, p.183-190, 2015.

El objetivo de este estudio fue evaluar in situ la reacción de 50 materiales genéticos de tres especies de Theobroma (T. cacao, T. grandiflorum y T. bicolor) a la inoculación controlada de tres aislados autóctonos de Moniliophthora roreri (agente causal de la moniliasis del cacao) en el Departamento de Caquetá (Amazonia colombiana). Las variables incidencia de la enfermedad (IMr), severidad externa (SE) y severidad interna (SI), se evaluaron nueve semanas después de la inoculación de mazorcas de 2 a 3 meses de edad con una suspensión de esporas de 1,2 × $10^{5}$ esporas $/ \mathrm{mL}$. La enfermedad se evidenció en las tres especies de Theobroma, sin embargo, ésta no se presentó en 13 de los 50 materiales genéticos evaluados, representados así: seis T. grandiflorum, cinco T. bicolor y dos T. cacao. Los materiales genéticos de T. bicolor y $T$. grandiflorum fueron los menos afectados comparados con los de T. cacao. Se encontraron diferencias significativas para IMr, SE y SI entre las tres especies de Theobroma y entre los 50 materiales genéticos. Diferencias significativas entre los aislados solo ocurrieron para SE. La incidencia y la severidad de la enfermedad entre materiales genéticos, fueron influenciadas por el patógeno aislado. Los materiales genéticos con menor reacción a M. roreri pueden ser utilizados en programas de mejoramiento por resistencia a la moniliasis.

Palabras claves: Moniliasis, Resistencia, incidencia, severidad

\section{RESUMO}

Sterling, C.A.; Hermida-Daza, M.A.; Rodriguez-León, C.H.; Salas-Tobón, Y. M.; Nieto-Guzmán, M.N.; Caicedo-Rodríguez, D.F. Reação de Theobroma spp. ao fungo Moniliophthora roreri em Caquetá, Colombia. Summa Phytopathologica, v.41, n.3, p.183-190, 2015.

O objetivo deste estudo foi avaliar in situ a reação de 50 materiais genéticos de três espécies de Theobroma (T. cacao, T. grandiflorum e T. bicolor) à inoculação controlada com três isolados autóctones de $M$. roreri (agente causal da monilíase do cacau) no Departamento de Caquetá (Amazônia colombiana). As variáveis incidência da doença (IMr), severidade externa (SE) e severidade interna (SI) foram avaliadas nove semanas após a inoculação de frutos de 2 a 3 meses de idade com una suspensão de esporos de $1,2 \times 10^{5}$ esporos $/ \mathrm{mL}$. A doença evidenciou-se nas três espécies de Theobroma, no entanto, 13 dos 50 materiais genéticos não apresentaram síntomas, bem representados: seis T. grandiflorum, cinco T. bicolor y dois T. cacao. Os genótipos de T. bicolor e T. grandiflorum foram os menos afetados em comparação aos de T. cacao. Encontraram-se diferenças significativas para IMr, SE e SI entre as três espécies de Theobroma e entre os 50 materiais genéticos. Diferenças significativas entre isolados só ocorreram para SE. A incidência e a severidade da doença entre materiais foram influenciadas pelo inóculo utilizado. Os genótipos com menor reação a $M$. roreri podem ser utilizados em programas de melhoramento para resistência à monilíase.

Palavras-chave: Monilíase, resistência, incidência, severidade.

ABSTRACT

Sterling, C.A.; Hermida-Daza, M.A.; Rodriguez-León, C.H.; Salas-Tobón, Y. M.; Nieto-Guzmán, M.N.; Caicedo-Rodríguez, D.F. Reaction to Moniliophthora roreri in Theobroma spp. at Caquetá, Colombia. Summa Phytopathologica, v.41, n.3, p.183-190, 2015.

The aim of this study was to evaluate in situ the reaction of 50 genetic materials of three species of Theobroma (T. cacao, T. grandiflorum and T. bicolor) to controlled inoculation of three autochthonous isolates of Moniliophtora roreri (the causal agent of moniliasis or frosty pod disease), at the Department of Caquetá (Colombian Amazonia). The variables disease incidence (IMr), external severity (SE), and internal severity (SI) were evaluated at nine weeks after inoculation of 2-to-3-month-old fruits with a spore suspension of $1.2 \times 10^{5}$ spores $/ \mathrm{mL}$. The disease was evidenced in the three species of Theobroma; however, 13 of the 50 genetic materials did not present symptoms, well represented by: six $T$. grandiflorum, five T. bicolor and two T. cacao. The genotypes of T. bicolor and $T$. grandiflorum were the least affected, compared to those of T. cacao. There were significant differences for IMr, SE and SI among the three species of Theobroma and among the 50 genetic materials. Significant differences among the isolates were found only for SE. The disease incidence and severity among materials were influenced by the used inoculum. Genotypes with less reaction to $M$. roreri can be used in breeding programs for the resistance to moniliasis.

Additional keywords: Moniliasis, resistence, incidence, severity. 


\section{INTRODUCCIÓN}

El género Theobroma comprende 22 especies y es exclusivamente neotropical. Éste se distribuye a lo largo de las selvas tropicales, desde los $18^{\circ}$ de latitud norte hasta los $15^{\circ}$ de latitud sur. Brasil y Colombia pueden ser considerados como los principales centros de diversidad de éste género, por albergar el mayor número de especies autóctonas (25). Entre las especies de Theobroma, el cacao común (Theobroma cacao L.), fuente del chocolate, es la especie más cultivada y económicamente importante, y en menor grado el copoazú (T. grandiflorum (Willd. ex Spreng.) K. Schum) y el maraco (T. bicolor Humb. \& Bonpl.), con diferentes usos principalmente en la industria agroalimentaria y cosmética (9).

La moniliasis, causada por el hongo Moniliophthora roreri (Cif \& Par.) Evans et al.), es la principal limitante del cultivo de cacao, principalmente en países como Ecuador, Costa Rica, Honduras, Perú, Colombia y México $(11,15,20)$.

Según Phillips-Mora et al. (18), la región norooriental de Colombia es considerada como el centro de mayor diversidad genética de $M$. roreri e hipotéticamente el centro de origen de la enfermedad. Se presume que la aparición de la enfermedad tiene relación con el proceso de domesticación del cacao en donde se involucró la asociación de éste con otras especies relacionadas como Theobroma gireli (18). En consecuencia, los géneros Theobroma y Herrania constituyeron hospederos naturales de M. roreri (7).

En Colombia, la moniliasis puede causar pérdidas del 40 al 100\% del grano seco de cacao dependiendo de las prácticas culturales (3). El control de $M$. roreri se basa principalmente en prácticas culturales, sin embargo, estas son complementadas con otros métodos debido al aumento de la agresividad del patógeno (18). Dentro de estos métodos se destacan el control biológico, químico y genético (19). El control genético es uno de los más promisorios ya que la selección de materiales genéticos resistentes podría proveer una alternativa exitosa a largo plazo y de bajo costo, integrada a otros métodos de manejo de la enfermedad $(11,20)$.

En Colombia, la selección de materiales genéticos regionales con características sobresalientes representa una de las principales estrategias para mejorar la productividad y la calidad del grano, y combatir la moniliasis (8). Sin embargo, en muchas zonas del país como la Amazonia colombiana, aún se siembran materiales vegetales introducidos que no han sido validados científicamente antes de ser recomendados al nivel del productor, lo que pone en riesgo el establecimiento de nuevas plantaciones que llegan a ser susceptibles a $M$. roreri (1).

Adicionalmente, en la Amazonia colombiana existe un desconocimiento del recurso genético regional tanto de $T$. cacao como de otras especies promisorias de Theobroma (T. grandiflorum y $T$. bicolor) (9). Esta situación representa una oportunidad para conocer y valorar el uso potencial de materiales genéticos autóctonos, adaptados a las condiciones edafoclimáticas de la región, con características agronómicas sobresalientes y con tolerancia o resistencia a los limitantes fitosanitarios, principalmente a la moniliasis.

El objetivo de este estudio fue evaluar la reacción in situ de 50 materiales genéticos de tres especies de Theobroma ( $T$. cacao, $T$. grandiflorum y $T$. bicolor) a la inoculación controlada de $M$. roreri en condiciones del departamento del Caquetá (Amazonia colombiana).

\section{MATERIALES Y MÉTODOS}

\section{Área de estudio}

Las pruebas de infección controlada, realizadas en campo con inóculo artificial de $M$. rorei, se llevaron a cabo en nueve municipios cacaoteros del departamento del Caquetá (Amazonia noroccidental Colombiana) (Tabla 1). El Caquetá presenta una precipitación promedio anual de $3245 \mathrm{~mm}$, una temperatura media de $25,4{ }^{\circ} \mathrm{C}$, la humedad relativa promedio es de $85,7 \%$ y el brillo solar medio de 1490 horas de luz/año (10).

\section{Fuente de inóculo}

Se emplearon tres aislados autóctonos de $M$. roreri: $\mathrm{MrC} 8$ (aislado 1), MrC20 (aislado 2) y MrC30 (aislado 3), obtenidos in vitro en el año 2012 a partir de frutos de T. cacao con síntomas y signos de moniliasis recolectados en tres municipios de tradición cacaotera (Florencia, El Doncello y Belén de los Andaquíes, respectivamente). Estos aislados se localizaron en el Cepario del Laboratorio de Fitopatología del Instituto Amazónico de Investigaciones Científicas Sinchi en Florencia, Caquetá. Los aislados se conservaron a temperatura ambiente $\left(24^{\circ} \mathrm{C}\right)$ en cajas de Petri de 5,2 cm de diámetro con medio agar extracto de malta enriquecido con jugo V8 durante 11 días y se sometieron a fotoperiodo 12 horas luz/oscuridad (20).

\section{Material genético}

Se utilizaron 50 materiales genéticos de tres especies de Theobroma (Tabla 1). La identidad del material genético fue confirmada por Munar et al. (16), mediante caracterización molecular utilizando la técnica SSR con 15 pares de cebadores microsatélites (mTcCIR3, mTcCIR6, mTcCIR9, mTcCIR10, mTcCIR11, mTcCIR12, mTcCIR13, mTcCIR17, mTcCIR18, mTcCIR19, mTcCIR21, mTcCIR22, mTcCIR25, mTcCIR26 y mTcCIR61).

\section{Inoculación}

Para las inoculaciones artificiales se utilizaron frutos de 2 a 3 meses de edad de acuerdo con la metodología descrita por Phillips-Mora (17). Previo a la inoculación, se preparó una suspensión de esporas para cada aislado mediante la adición de $20 \mathrm{~mL}$ de agua destilada a cada caja de Petri y se removieron las esporas con un pincel. Se utilizaron dos cajas de Petri por cada aislado. La suspensión se diluyó con $200 \mathrm{~mL}$ de agua destilada estéril y dos gotas de Tween 80 . La mezcla se agitó durante 10 minutos y luego se ajustó la concentración de esporas a $1,2 \times 10^{5}$ esporas/mL de acuerdo con el método de Sánchez et al. (26).

Las inoculaciones se realizaron con un bulbo atomizador a través del cual se aplicaron tres aspersiones distribuidas homogéneamente sobre los frutos seleccionados. Luego las mazorcas se cubrieron con bolsas trasparentes de polietileno en las que se depositó una toalla de papel estéril humedecida con $30 \mathrm{~mL}$ de agua destilada estéril con el fin de proveer una cámara húmeda requerida para favorecer el proceso germinativo e infeccioso del patógeno. Se inocularon entre dos y tres frutos por cada aislado en cada material de Theobroma y se realizaron cinco réplicas de inoculación entre los años 2012 y 2013. El número de réplicas reales realizadas en cada material estuvo relacionado con la disponibilidad de frutos presentes en el árbol al momento de la inoculación.

\section{Evaluación}

La evaluación de la enfermedad se realizó nueve semanas después de la inoculación. Se evaluaron las variables: 1) incidencia de la enfermedad (IMr) como el porcentaje de frutos enfermos en relación 


\begin{tabular}{|c|c|c|}
\hline Especie de Theobroma & Material genetico & Procedencia \\
\hline \multirow{8}{*}{ T. cacao (Cacao común) } & SCSA* 97, 103 & El Paujil ${ }^{1}$ \\
\hline & SCSA 104,108 & Valparaiso $^{2}$ \\
\hline & SCSA 16,18 & San Vicente del Caguan ${ }^{3}$ \\
\hline & SCSA $27,28,29,30$ & Puerto Rico ${ }^{4}$ \\
\hline & SCSA $42,43,45,50,71$ & Florencia $^{5}$ \\
\hline & SCSA $140,142,151$ & Belen de los Andaquíes ${ }^{6}$ \\
\hline & SCSA $3,5,6,75,88,90$ & El Doncello ${ }^{7}$ \\
\hline & SCSA $107,174,175,177,178,179,180$ & Albania $^{8}$ \\
\hline \multirow{4}{*}{ T. grandiflorum (Copoazú) } & SCSA 73 & El Doncello $^{7}$ \\
\hline & SCSA $152,154,158$ & Belen de los Andaquíes ${ }^{6}$ \\
\hline & SCSA $160,161,169,170$ & Albania $^{8}$ \\
\hline & SCSA $115,117,121,124$ & Valparaiso $^{2}$ \\
\hline \multirow{4}{*}{ T. bicolor (Bacao ó Maraco) } & $\begin{array}{l}\text { SCSA } 64 \\
\text { SCSA } 41\end{array}$ & $\begin{array}{l}\text { Florencia }^{5} \\
\text { La Montañita }^{9}\end{array}$ \\
\hline & SCSA 32 & El Doncello ${ }^{7}$ \\
\hline & SCSA 143 & Belen de los Andaquíes ${ }^{6}$ \\
\hline & SCSA $113,119,120$ & Valparaiso $^{2}$ \\
\hline
\end{tabular}

*SCSA Selección Caquetá Sinchi Acamafrut

${ }^{1} 1^{\circ} 34^{\prime} 10^{\prime \prime} \mathrm{N}, 75^{\circ} 19^{\prime} 35^{\prime \prime} \mathrm{W} ;^{2} 1^{\circ} 11^{\prime} 36^{\prime \prime} \mathrm{N}, 75^{\circ} 42^{\prime} 23^{\prime \prime} \mathrm{W} ;{ }^{3} 2^{\circ} 6^{\prime} 55^{\prime \prime} \mathrm{N}, 74^{\circ} 46^{\prime} 12^{\prime \prime} \mathrm{W} ;^{4} 1^{\circ} 54^{\prime} 27^{\prime \prime} \mathrm{N}, 75^{\circ} 9^{\prime} 30^{\prime \prime} \mathrm{W} ;{ }^{5} 1^{\circ} 36^{\prime} 51^{\prime \prime} \mathrm{N}, 75^{\circ} 36^{\prime} 42^{\prime \prime} \mathrm{W} ;{ }^{6} 1^{\circ} 24^{\prime}$ $59^{\prime \prime} \mathrm{N}, 75^{\circ} 52^{\prime} 21^{\prime \prime} \mathrm{W} ;^{7} 1^{\circ} 40^{\prime} 48^{\prime \prime} \mathrm{N}, 75^{\circ} 17^{\prime} 6^{\prime \prime} \mathrm{W} ;^{8} 1^{\circ} 19^{\prime} 44^{\prime \prime} \mathrm{N}, 75^{\circ} 52^{\prime} 42^{\prime \prime} \mathrm{W} ;^{9} 1^{\circ} 28^{\prime} 45^{\prime \prime} \mathrm{N}, 75^{\circ} 26^{\prime} 10^{\prime \prime} \mathrm{W}$.

Tabla 1. Materiales genéticos de Theobroma spp. probados por la reacción a Moniliophthora roreri en Caquetá, Colombia.

con el total de frutos inoculados; 2) severidad externa (SE): basada en la apariencia externa del fruto y los signos del patógeno, mediante la escala propuesta por Sánchez et al. (26), donde: grado $0=$ fruto sano, $1=$ puntos aceitosos; $2=$ hinchazón y/o maduración prematura; $3=$ mancha (necrosis); $4=$ micelio hasta un $25 \%$ de la mancha necrótica; $5=$ micelio que cubre más del $25 \%$ de la mancha necrótica. Esta variable mide el nivel de daño externo causado por el hongo y su habilidad para producir propágulos. 3) severidad interna (SI): basada en el porcentaje de necrosis interna observada en el fruto cuando es cortado longitudinalmente y medido con relación a la escala desarrollada por Sánchez et al. (26), donde: grado $0=0 \%$ área necrosada; $1=1-20 \%$ del área necrosada; $2=21-40 \%$ área necrosada; $3=41-60 \%$ área necrosada; 4=61-80\% área necrosada; $5=>80 \%$ área necrosada.

El tipo de reacción a $M$. roreri de los materiales genéticos de Theobroma se clasificó por la media de los valores de SI a partir de la escala adaptada de Phillips-Mora et al. (19): Resistente $(0-1,25)$, moderadamente resistente $(1,26-2,50)$, moderadamente susceptible $(2,51-3,75)$ y susceptible $(3,76-5,0)$.

\section{Análisis de resultados}

Las variables estudiadas se analizaron mediante una ANOVA con prueba de Tukey al 5\% y un análisis de correlación múltiple de Pearson $(\alpha=0,05)$. Adicionalmente se analizaron las variables asociadas a la reacción artificial mediante un análisis de componentes principales (ACP) para los 50 materiales genéticos y un análisis discriminante para las tres especies de Theobroma. Las pruebas estadísticas se corrieron en el programa InfoStat versión 2013 (5).

\section{RESULTADOS Y DISCUSION}

Se evidenció la enfermedad en las tres especies de Theobroma y hubo ausencia de la misma en el $26 \%$ de los materiales genéticos (Tabla 2). Debido a que los aislados utilizados en las inoculaciones controladas, procedían de frutos de T. cacao infectado por el hongo $M$. roreri, los materiales genéticos de $T$. bicolor y $T$. grandiflorum fueron los menos afectados comparados con los materiales genéticos de T. cacao en donde se presentaron las mayores incidencias y notas de severidad de la enfermedad (Tablas 2 y 3). La mayor correlación se presentó entre SE y SI $(r=0,94, \mathrm{gl}=207, P<0,05)$ y las menores correlaciones se observaron entre IMr y la severidad $(r=0,85, \mathrm{gl}=207$, $P<0,05$ respecto a SE y $r=0.82, \mathrm{gl}=207, P<0,05$, en relación con $\mathrm{SI})$.

El análisis de componentes principales (ACP) realizado sobre la matriz de correlación mostró que los dos primeros componentes aportaron el $86 \%$ de la variación total y presentaron raíces características mayores a la unidad (Figura 1). Al analizar los coeficientes de los vectores característicos asociados a los dos primeros componentes, se concluyó que las variables mostraron el siguiente orden de importancia: SE1, SE3, SI1, IMr1, IMr3 y SI3. Es decir, que la mayor variabilidad en la expresión de la enfermedad entre los 50 materiales genéticos se debió principalmente al efecto producido por los aislados $\mathrm{MrC} 8$ y $\mathrm{MrC} 30$ de $M$. roreri.

El ACP por materiales genéticos permitió evidenciar seis grupos (Figura 1). En el grupo A se encuentran los 25 materiales genéticos que reaccionaron a los tres aislados de $M$. roreri y presentaron las mayores notas de incidencia y severidad. En el grupo B se localizaron los 13 materiales genéticos que no presentaron síntomas de la enfermedad. En el grupo C se ubicaron los cuatro materiales genéticos que sólo reaccionaron positivamente a los aislados $\mathrm{MrC} 8$ y $\mathrm{MrC} 30$. En el grupo 


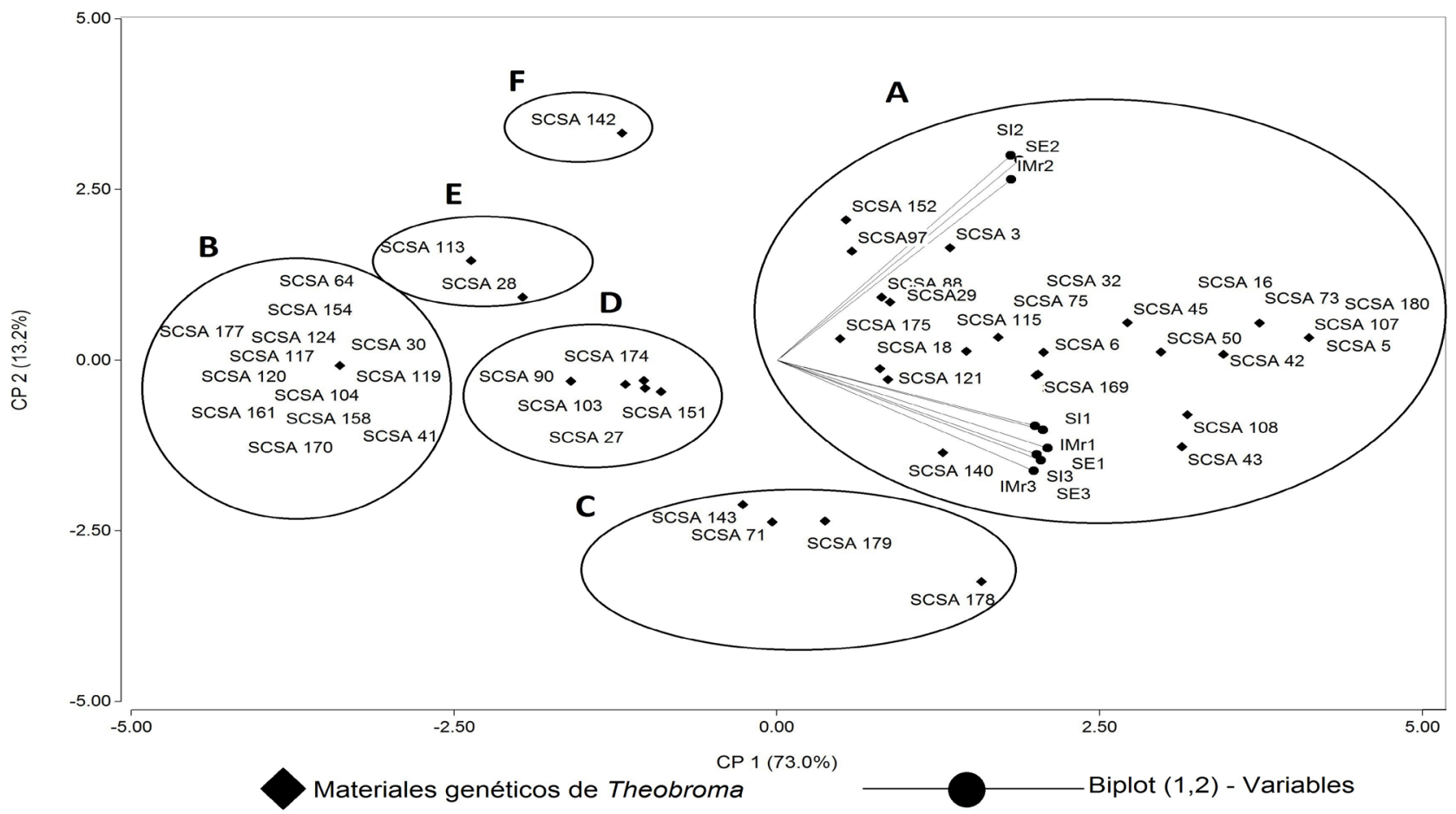

Figura 1. Análisis de componentes principales (gráfico biplot) de nueve variables combinadas (IMr, SE y SI x tres aislados) asociadas la reacción a $M$. roreri de 50 materiales del género Theobroma seleccionados en Caquetá (Colombia). Clave: IMr1, SE1 y SI1; IMr2, SE2 y SI2; IMr3, SE3 y SI3: Incidencia (IMr), severidad externa (SE) y severidad interna (SI) producidas por los aislados de M. roreri $\mathrm{MrC} 8$ (1), MrC20 (2) y MrC30 (3), respectivamente.

D se localizaron los cinco materiales genéticos que expresaron notas intermedias debido a la reacción positiva frente a los tres aislados probados. En el grupo E se ubicaron los dos materiales genéticos SCSA 28 y SCSA 113 que reaccionaron positivamente con notas bajas de severidad a los aislados $\mathrm{MrC} 8(\mathrm{SE}=1,0 ; \mathrm{SI}=0,6)$ y $\mathrm{MrC} 20(\mathrm{SE}=1,7$; $\mathrm{SI}=1,3)$ respectivamente. El grupo $\mathrm{F}$ que correspondió al material genético SCSA 142 reaccionó exclusivamente al aislado $\mathrm{MrC} 20$ con una nota media de 4,0, por lo resultó ser susceptible a la enfermedad.

El análisis discriminante (AD) realizado sobre los grupos identificados a priori (tres especies de Theobroma) mostró que con sólo dos ejes canónicos se tiene el 100\% de la discriminación entre grupos, donde el primer eje posee el 95,2\% del poder discriminatorio (Figura 2).

Según la figura 2, los síntomas en $T$. cacao correspondieron principalmente a daños internos (SI) en el fruto ocasionados por los tres aislados de $M$. roreri. En $T$. grandiflorum los síntomas internos se debieron principalmente al aislado $2(\mathrm{MrC20})$ y en $T$. bicolor los síntomas más característicos ocurrieron a nivel externo principalmente por los aislados 1 (MrC8) y 2 ( $\mathrm{MrC} 20)$.

Los resultados de éste estudio coinciden con lo reportado por (17, $19,22)$ quienes encontraron una mayor incidencia de la enfermedad asociada a una mayor severidad de la misma y un patrón diferencial en la expresión de los síntomas debido al tipo de aislado utilizado (Figuras 1 y 2). Estos autores también afirmaron que la intensidad de los daños internos se correlacionó positivamente con los síntomas externos expresados en el fruto. Sin embargo, los resultados de este estudio difieren de lo reportado por CATIE (4) en donde no se presentó correlación entre la severidad interna y externa, pero si entre la severidad interna y la incidencia de la enfermedad.

\section{Incidencia de $M$. roreri (IMr)}

Se encontraron diferencias significativas para IMr entre las tres especies de Theobroma y entre los 50 materiales genéticos (ambos $P<0,05)$. No se encontraron diferencias entre los tres aislados de M. roreri $(P>0,05)$ (Tabla 2). Por otro lado, la interacción material genético*aislado resultó significativa $(P<0,05)$, es decir, que la incidencia de la enfermedad entre materiales genéticos fue influenciada por el aislado.

El valor medio de la IMr fue de $56,9 \%$ en los 50 materiales genéticos evaluados, con un rango que oscilo entre 0 en el $26 \%$ de los materiales genéticos y $100 \%$ de la enfermedad en el $28 \%$ de los materiales genéticos evaluados $(P<0,05)$ (Tabla 2$)$. Entre especies el valor promedio de IMr osciló entre $28,57 \%$ para $T$. bicolor y $72,60 \%$ en $T$. cacao $(P<0,05)$.

Los resultados de este estudio, contrastan con lo reportado por Porras (23) quien no encontró diferencias significativas en la incidencia de la enfermedad entre seis cultivares de T. cacao evaluados. Sin embargo, los resultados de esta investigación en relación en los casos que se presentaron bajos valores medios de IMr, coinciden con lo reportado por Melgar et al. (15) quien encontró incidencias bajas ( 0 a $0,9 \%$ ) en clones regionales de Honduras.

En el caso de los materiales genéticos de cacao que exhibieron altas incidencias, estos resultados coinciden con lo reportado por $(2$, $13,17)$. No obstante, $(12,15,19,22)$ reportaron incidencias mayores al $60 \%$, las cuales resultaron superiores a la incidencia media reportada en este estudio.

Es importante destacar que la incidencia es un parámetro que puede contribuir con la identificación de materiales genéticos con buenas características en cuanto a su reacción a $M$. roreri. Estas características deben involucrar mecanismos de resistencia poligénica que impidan o 


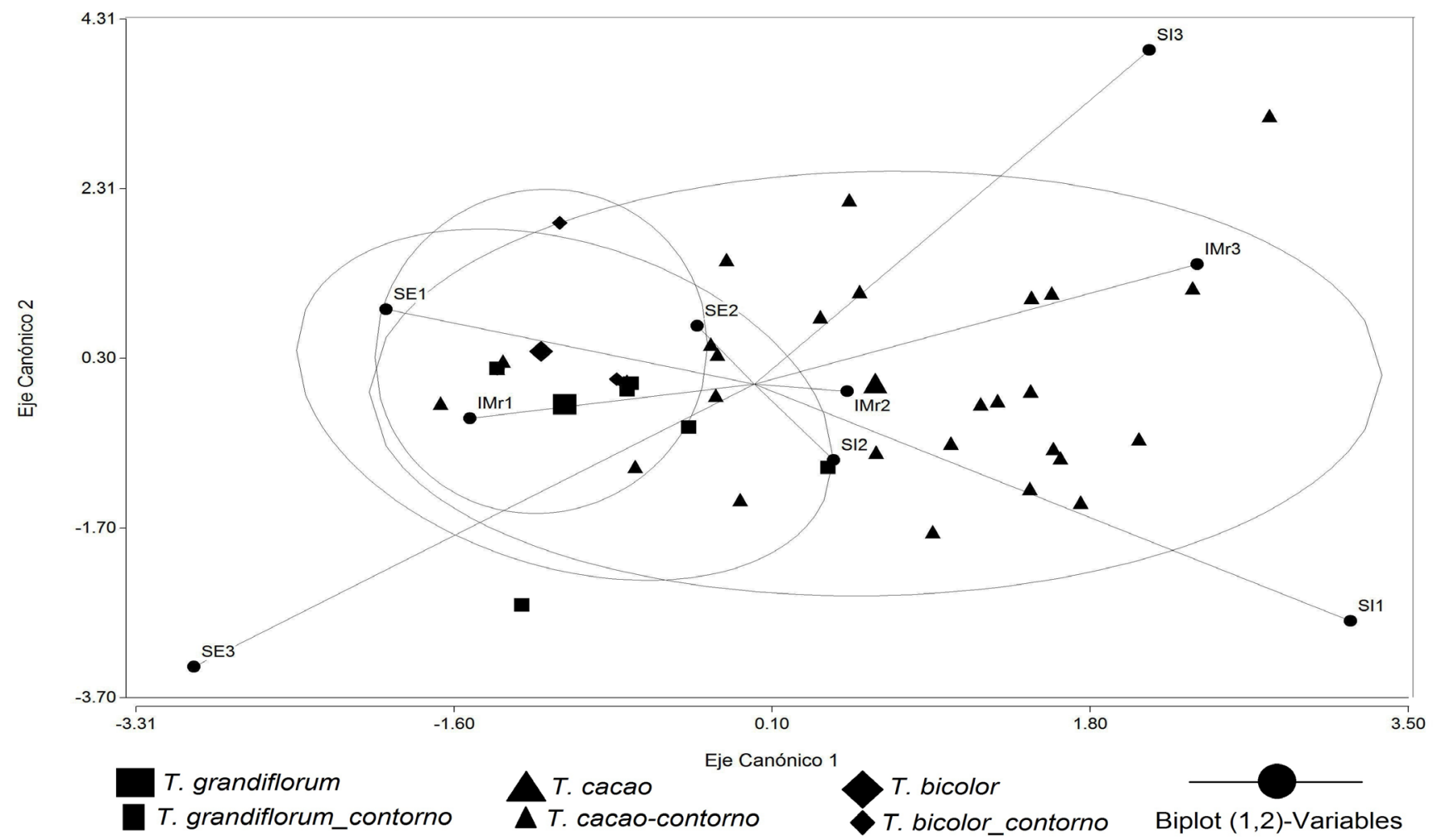

Figura 2. Gráfico biplot con elipses de predicción al 90\% representando los dos primeros ejes canónicos obtenidos mediante un análisis discriminante de tres grupos conocidos a priori (T. cacao, T. grandiflorum y T. bicolor) usando nueve variables combinadas (IMr, SE y SI x tres aislados) asociadas la reacción in situ a $M$. roreri en Caquetá (Colombia). Clave: IMr1, SE1 y SI1; IMr2, SE2 y SI2; IMr3, SE3 y SI3: Incidencia (IMr), severidad externa (SE) y severidad interna (SI) producidas por los aislados de M. roreri MrC8 (1), MrC20 (2) y MrC30 (3), respectivamente.

retrasen el desarrollo del patógeno (19).

\section{Severidad externa (SE)}

Se encontraron diferencias significativas en el valor medio de SE entre las tres especies de Theobroma, en los 50 materiales genéticos evaluados y entre los tres aislados de $M$. roreri (todos, $P<0,05$ ) (Tabla $3)$. La interacción material genético*aislado resultó significativa $(P<$ $0,05)$, es decir, que la variación del valor medio para SE entre materiales genéticos fue afectada por el aislado.

Según la Tabla 3, el valor medio de SE fue de 2,0 el cual osciló entre 0 en el $26 \%$ de los materiales genéticos y 5,0 en los materiales genéticos de T. cacao SCSA 5, SCSA 180 y SCSA $107(P<0,05)$, los cuales presentaron un micelio blanquecino que cubrió más del $25 \%$ de la mancha chocolate presente en los frutos inoculados. El aislado MrC8 fue el más agresivo, ya que generó síntomas en el 68\% de los materiales genéticos. El valor medio de SE entre especies osciló entre 0,73 para $T$. bicolor y 2,46 en $T$. cacao $(P<0,05)$.

Los resultados de este estudio para SE, coinciden con lo reportado por $(12,14,15,17,22)$ quienes reportaron el desarrollo de la enfermedad en frutos de cacao hasta la formación de la mancha necrótica con esporulación.

No obstante, los resultados de esta investigación son contrarios a lo encontrado por Porras (23) quien registró los síntomas externos débiles en los clones comerciales de cacao evaluados. Las diferencias en el tipo de reacción están relacionadas no sólo por el genotipo del material sino también por la virulencia del aislado probado (19).

Evans (6) establece que la SE no permite intuir con precisión el desarrollo de infecciones internas en el endocarpo de los frutos de cacao. Así la SE no determina la resistencia de los materiales genéticos pero si aporta información sobre la tolerancia de los frutos al inóculo de $M$. roreri $(19,27)$. Ribeiro et al. (24) afirman que factores como el número y la densidad del inóculo pueden ocasionar un incremento o una disminución de los daños expresados externamente por los frutos.

\section{Severidad interna (SI)}

Se encontraron diferencias significativas para SI entre las tres especies de Theobroma, y en los 50 materiales genéticos evaluados $(P$ $<0,05)$. Así mismo, la interacción material genético*aislado resultó significativa $(\mathrm{P}<0,05)$, lo que significa que las diferencias observadas entre los tres aislados fue influenciada por el material genético inoculado, similar a lo que se observó en las variables IMr y SE.

Según la Tabla 3 el valor medio de SI fue de 1,96 con un rango que osciló entre 0 en el 26\% de los materiales genéticos y 5,0 para las selecciones de T. cacao SCSA 5, SCSA 180 y SCSA $107(P<0,05)$. Entre especies de Theobroma SI osciló entre 0,56 para la especie $T$. bicolor y 2,52 para T. cacao $(P<0,05)$.

Se observó además que los aislados $\mathrm{MrC} 8$ y MrC30 fueron los más agresivos, ya que generaron síntomas necróticos internos en el $66 \%$ de los materiales genéticos. Independiente del aislado probado, se observó que el $16 \%$ de los materiales genéticos presentaron una SI con notas medias superiores a 4, lo que indica un daño necrótico del 60 al 100\% de las almendras presentes en los frutos (Tabla 3).

En este estudio, las diferencias significativas encontradas en la SI entre materiales genéticos coinciden con lo registrado por $(17,19$, 


\begin{tabular}{|c|c|c|c|c|c|c|c|}
\hline Factor & Nivel & Especie & IMr & Factor & Nivel & Especie & IMr \\
\hline \multirow{24}{*}{$\begin{array}{l}\text { Material } \\
\text { genético }\end{array}$} & SCSA 154 & T. grandiflorum & $0,0 \pm 15,0^{a} b^{b}$ & \multirow{24}{*}{$\begin{array}{l}\text { Material } \\
\text { genético }\end{array}$} & SCSA 152 & T. grandiflorum & $66,7 \pm 15,0 \mathrm{ab}$ \\
\hline & SCSA 158 & T. grandiflorum & $0,0 \pm 15,0 b$ & & SCSA 121 & T. grandiflorum & $66,7 \pm 15,0 \mathrm{ab}$ \\
\hline & SCSA 120 & T. bicolor & $0,0 \pm 15,0 b$ & & SCSA 179 & T. сасаo & $66,7 \pm 15,0 \mathrm{ab}$ \\
\hline & SCSA 104 & T. cacao & $0,0 \pm 15,0 b$ & & SCSA 3 & T. cacao & $66,7 \pm 15,0 \mathrm{ab}$ \\
\hline & SCSA 161 & T. grandiflorum & $0,0 \pm 15,0 b$ & & SCSA 88 & T. сасаo & $68,5 \pm 15,0 \mathrm{ab}$ \\
\hline & SCSA 177 & T. сасаo & $0,0 \pm 15,0 b$ & & SCSA 6 & T. cacao & $83,3 \pm 15,0 \mathrm{ab}$ \\
\hline & SCSA 170 & T. grandiflorum & $0,0 \pm 15,0 b$ & & SCSA 140 & T. сасаo & $83,3 \pm 15,0 \mathrm{ab}$ \\
\hline & SCSA 117 & T. grandiflorum & $0,0 \pm 15,0 b$ & & SCSA 108 & T. cacao & $88,9 \pm 15,0 \mathrm{a}$ \\
\hline & SCSA 119 & T. bicolor & $0,0 \pm 15,0 b$ & & SCSA 16 & T. сасаo & $94,4 \pm 15,0 a$ \\
\hline & SCSA 30 & T. cacao & $0,0 \pm 15,0 b$ & & SCSA 45 & T. cacao & $94,4 \pm 15,0 \mathrm{a}$ \\
\hline & SCSA 124 & T. grandiflorum & $0,0 \pm 15,0 b$ & & SCSA 5 & T. сасаo & $100,0 \pm 15,0 \mathrm{a}$ \\
\hline & SCSA 41 & T. bicolor & $0,0 \pm 15,0 b$ & & SCSA 73 & T. grandiflorum & $100,0 \pm 15,0 \mathrm{a}$ \\
\hline & SCSA 28 & T. cacao & $27,8 \pm 15,0 \mathrm{ab}$ & & SCSA 42 & T. сасаo & $100,0 \pm 15,0 \mathrm{a}$ \\
\hline & SCSA 142 & T. cacao & $33,3 \pm 15,0 \mathrm{ab}$ & & SCSA 180 & T. cacao & $100,0 \pm 15,0 \mathrm{a}$ \\
\hline & SCSA 90 & T. cacao & $33,3 \pm 15,0 a b$ & & SCSA 115 & T. grandiflorum & $100,0 \pm 15,0 \mathrm{a}$ \\
\hline & SCSA 113 & T. bicolor & $33,3 \pm 15,0 a b$ & & SCSA 160 & T. grandiflorum & $100,0 \pm 15,0 \mathrm{a}$ \\
\hline & SCSA 71 & T. cacao & $44,4 \pm 15,0 \mathrm{ab}$ & & SCSA 169 & T. grandiflorum & $100,0 \pm 15,0 \mathrm{a}$ \\
\hline & SCSA 103 & T. cacao & $49,1 \pm 15,0 \mathrm{ab}$ & & SCSA 18 & T. cacao & $100,0 \pm 15,0 \mathrm{a}$ \\
\hline & SCSA 174 & T. сасао & $50,0 \pm 15,0 \mathrm{ab}$ & & SCSA 50 & T. сасаo & $100,0 \pm 15,0 \mathrm{a}$ \\
\hline & SCSA 27 & T. cacao & $63,9 \pm 15,0 \mathrm{ab}$ & & SCSA 43 & T. cacao & $100,0 \pm 15,0 \mathrm{a}$ \\
\hline & SCSA 175 & T. cacao & $64,8 \pm 15,0 \mathrm{ab}$ & & SCSA 29 & T. сасао & $100,0 \pm 15,0 \mathrm{a}$ \\
\hline & SCSA 143 & T. bicolor & $66,7 \pm 15,0 \mathrm{ab}$ & & SCSA 75 & T. cacao & $100,0 \pm 15,0 \mathrm{a}$ \\
\hline & SCSA 151 & T. cacao & $66,7 \pm 15,0 \mathrm{ab}$ & & SCSA 107 & T. сасао & $100,0 \pm 15,0 \mathrm{a}$ \\
\hline & SCSA 97 & T. cacao & $66,7 \pm 15,0 \mathrm{ab}$ & & SCSA 32 & T. bicolor & $100,0 \pm 15,0 \mathrm{a}$ \\
\hline \multicolumn{2}{|c|}{ Factor } & \multicolumn{2}{|c|}{ Nivel } & \multicolumn{2}{|c|}{ Especie } & \multicolumn{2}{|c|}{ IMr } \\
\hline \multirow{4}{*}{\multicolumn{2}{|c|}{ Aislado }} & \multicolumn{2}{|c|}{$\mathrm{IMrC}^{\mathrm{c}}$} & \multirow{4}{*}{\multicolumn{2}{|c|}{ M. roreri }} & \multicolumn{2}{|c|}{$56,2 \pm 3,7 \mathrm{a}$} \\
\hline & & \multirow{2}{*}{\multicolumn{2}{|c|}{$\begin{array}{l}\mathrm{IMrC} 20 \\
\mathrm{IMrC} 30\end{array}$}} & & & \multirow{2}{*}{\multicolumn{2}{|c|}{$\begin{array}{l}56,8 \pm 3,7 a \\
57,7 \pm 3,7 a\end{array}$}} \\
\hline & & & & & & & \\
\hline & & \multicolumn{2}{|c|}{ Media } & & & \multicolumn{2}{|c|}{56,9} \\
\hline
\end{tabular}

${ }^{a}$ Error estandar; ${ }^{b}$ Valores en cada columna seguidos por la misma letra no difieren estadísticamente (Prueba Tukey HSD, P $\left.<0,05\right)$; ${ }^{\mathrm{c}}$ Aislados de M. roreri (IMrC8), (IMrC20) y (IMrC30)

Tabla 2. Medias de incidencia (\%) de moniliasis producida por la inoculación controlada de M. roreri en 50 materiales genéticos de Theobroma (T. cacao, T. grandiflorum y T. bicolor) seleccionados en Caquetá, Colombia.

22). Del mismo modo, la ausencia de significancia observada entre los tres aislados probados en esta investigación coincide con lo reportado Phillips-Mora et al. (19) quienes no encontraron diferencias en la SI producida por siete cepas evaluadas en cinco clones de interés comercial en Santander, Colombia.

Las menores notas de SI en este estudio, fueron similares a lo encontrado por $(12,15)$ quienes reportaron notas de SI con un rango de 0,90 a 1,63, es decir, daños internos en el 16\% de las almendras.

Las mayores notas de SI en este estudio ( $>4)$, fueron similares a lo encontrado por $(4,19,21,23)$ quienes reportaron notas de SI que oscilaron entre 2,3 y 5 , es decir, con un necrosamiento interno entre el $60-100 \%$.

A partir de éste estudio se pudo concluir que: a) de los 50 materiales genéticos evaluados, el $44 \%$ presentaron resistencia completa o moderada a $M$. roreri y el $26 \%$ no presentaron signos ni síntomas de la enfermedad (cuatro materiales genéticos de $T$. bicolor, tres de $T$. cacao y seis de $T$. grandiflorum); b) los materiales genéticos con resistencia a $M$. roreri deben evaluarse en experimentos clonales a gran escala antes de ofrecer una recomendación final al nivel del productor; c) este es el primer estudio en el que se evalúa el tipo de reacción in situ a inoculación controlada de M. roreri de las especies $T$. bicolor y $T$. grandiflorum.

\section{AGRADECIMIENTOS}

Los autores agradecen al proyecto "Selección y evaluación in situ de árboles élites del género Theobroma, como estrategia de rescate y aprovechamiento del germoplasma local con potencial para la Amazonia colombiana"- Contrato RC No.628 -2011- Fiduciara Bogotá - Colciencias - Sinchi por el soporte económico a la presente investigación. 


\begin{tabular}{|c|c|c|c|c|c|c|c|c|c|c|c|c|}
\hline \multirow{3}{*}{$\begin{array}{l}\text { Material } \\
\text { ge nético }\end{array}$} & \multirow{3}{*}{ Especie } & \multicolumn{4}{|c|}{ SE } & \multicolumn{7}{|c|}{ SI } \\
\hline & & \multicolumn{4}{|c|}{ Aislado } & \multicolumn{7}{|c|}{ Aislado } \\
\hline & & MrC8 & MrC20 & MrC30 & Promedio & MrC8 & $\mathbf{T R}^{\mathrm{a}}$ & MrC20 & TR & MrC30 & TR & $\overline{\text { Promedio }}$ \\
\hline SCSA 97 & T. cacao & $0,0 \pm 0,5^{\mathrm{b}} \mathrm{a}^{\mathrm{c}}$ & $3,0 \pm 5,0 \mathrm{a}-\mathrm{h}$ & $3,0 \pm 0,5 \mathrm{a}-\mathrm{h}$ & $2,0 \mathrm{~b}-\mathrm{g}$ & $0,0 \pm 0,5 \mathrm{a}$ & $\mathrm{R}$ & $5,0 \pm 0,5 \mathrm{i}$ & MS & $4,0 \pm 0,5 \mathrm{e}-\mathrm{i}$ & MS & $3,0 \mathrm{~h}-\mathrm{k}$ \\
\hline SCSA 117 & T. grandiflorum & $0,0 \pm 0,5 \mathrm{a}$ & $0,0 \pm 0,5 \mathrm{a}$ & $0,0 \pm 0,5 \mathrm{a}$ & $0,0 \mathrm{a}$ & $0,0 \pm 0,5 \mathrm{a}$ & $\mathrm{R}$ & $0,0 \pm 0,5 \mathrm{a}$ & $\mathrm{R}$ & $0,0 \pm 0,5 \mathrm{a}$ & $\mathrm{R}$ & $0,0 \mathrm{a}$ \\
\hline SCSA 30 & T. cacao & $0,0 \pm 0,4 \mathrm{a}$ & $0,0 \pm 0,4 \mathrm{a}$ & $0,0 \pm 0,5 \mathrm{a}$ & $0,0 \mathrm{a}$ & $0,0 \pm 0,4 a$ & $\mathrm{R}$ & $0,0 \pm 0,4 \mathrm{a}$ & $\mathrm{R}$ & $0,0 \pm 0,5 \mathrm{a}$ & $\mathrm{R}$ & $0,0 \mathrm{a}$ \\
\hline SCSA 113 & T. bicolor & $0,0 \pm 0,5 \mathrm{a}$ & $1,0 \pm 0,5 \mathrm{ae}$ & $0,0 \pm 0,5 \mathrm{a}$ & $0,3 \mathrm{ab}$ & $0,0 \pm 0,5 a$ & $\mathrm{R}$ & $1,0 \pm 0,5 \mathrm{a}-\mathrm{d}$ & $\mathrm{R}$ & $0,0 \pm 0,5 \mathrm{a}$ & $\mathrm{R}$ & $0,3 \mathrm{ab}$ \\
\hline SCSA 124 & T. grandiflorum & $0,0 \pm 0,5 \mathrm{a}$ & $0,0 \pm 0,5 \mathrm{a}$ & $0,0 \pm 0,5 \mathrm{a}$ & $0,0 \mathrm{a}$ & $0,0 \pm 0,5 \mathrm{a}$ & $\mathrm{R}$ & $0,0 \pm 0,5 \mathrm{a}$ & $\mathrm{R}$ & $0,0 \pm 0,5 \mathrm{a}$ & $\mathrm{R}$ & $0,0 \mathrm{a}$ \\
\hline SCSA 119 & T. bicolor & $0,0 \pm 0,5 \mathrm{a}$ & $0,0 \pm 0,5 \mathrm{a}$ & $0,0 \pm 0,5 \mathrm{a}$ & $0,0 \mathrm{a}$ & $0,0 \pm 0,5 \mathrm{a}$ & $\mathrm{R}$ & $0,0 \pm 0,5 \mathrm{a}$ & $\mathrm{R}$ & $0,0 \pm 0,5 \mathrm{a}$ & $\mathrm{R}$ & $0,0 \mathrm{a}$ \\
\hline SCSA 120 & T. bicolor & $0,0 \pm 0,5 \mathrm{a}$ & $0,0 \pm 0,5 \mathrm{a}$ & $0,0 \pm 0,5 \mathrm{a}$ & $0,0 \mathrm{a}$ & $0,0 \pm 0,5 \mathrm{a}$ & $\mathrm{R}$ & $0,0 \pm 0,5 \mathrm{a}$ & $\mathrm{R}$ & $0,0 \pm 0,5 \mathrm{a}$ & $\mathrm{R}$ & $0,0 \mathrm{a}$ \\
\hline SCSA 142 & T.cacao & $0,0 \pm 0,5 \mathrm{a}$ & $5,0 \pm 0,5 \mathrm{~h}$ & $0,0 \pm 0,5 \mathrm{a}$ & $1,7 \mathrm{a}-\mathrm{f}$ & $0,0 \pm 0,5 \mathrm{a}$ & $\mathrm{R}$ & $4,0 \pm 0,5 \mathrm{e}-\mathrm{i}$ & S & $0,0 \pm 0,5 \mathrm{a}$ & $\mathrm{R}$ & $1,3 a-g$ \\
\hline SCSA 154 & T. grandiflorum & $0,0 \pm 0,5 \mathrm{a}$ & $0,0 \pm 0,5 \mathrm{a}$ & $0,0 \pm 0,5 \mathrm{a}$ & $0,0 \mathrm{a}$ & $0,0 \pm 0,5 \mathrm{a}$ & $\mathrm{R}$ & $0,0 \pm 0,5 \mathrm{a}$ & $\mathrm{R}$ & $0,0 \pm 0,5 \mathrm{a}$ & $\mathrm{R}$ & $0,0 \mathrm{a}$ \\
\hline SCSA 158 & T. grandiflorum & $0,0 \pm 0,5 \mathrm{a}$ & $0,0 \pm 0,5 \mathrm{a}$ & $0,0 \pm 0,5 \mathrm{a}$ & $0,0 \mathrm{a}$ & $0,0 \pm 0,5 \mathrm{a}$ & $\mathrm{R}$ & $0,0 \pm 0,5 \mathrm{a}$ & $\mathrm{R}$ & $0,0 \pm 0,5 \mathrm{a}$ & $\mathrm{R}$ & $0,0 \mathrm{a}$ \\
\hline SCSA 104 & T. cacao & $0,0 \pm 0,5 \mathrm{a}$ & $0,0 \pm 0,5 \mathrm{a}$ & $0,0 \pm 0,4 \mathrm{a}$ & $0,0 \mathrm{a}$ & $0,0 \pm 0,5 a$ & $\mathrm{R}$ & $0,0 \pm 0,5 \mathrm{a}$ & $\mathrm{R}$ & $0,0 \pm 0,4 \mathrm{a}$ & $\mathrm{R}$ & $0,0 \mathrm{a}$ \\
\hline SCSA 161 & T. grandiflorum & $0,0 \pm 0,5 \mathrm{a}$ & $0,0 \pm 0,5 \mathrm{a}$ & $0,0 \pm 0,5 \mathrm{a}$ & $0,0 \mathrm{a}$ & $0,0 \pm 0,5 \mathrm{a}$ & $\mathrm{R}$ & $0,0 \pm 0,5 \mathrm{a}$ & $\mathrm{R}$ & $0,0 \pm 0,5 \mathrm{a}$ & $\mathrm{R}$ & $0,0 \mathrm{a}$ \\
\hline SCSA 170 & T. grandiflorum & $0,0 \pm 0,5 \mathrm{a}$ & $0,0 \pm 0,5 \mathrm{a}$ & $0,0 \pm 0,5 \mathrm{a}$ & $0,0 \mathrm{a}$ & $0,0 \pm 0,5 \mathrm{a}$ & $\mathrm{R}$ & $0,0 \pm 0,5 \mathrm{a}$ & $\mathrm{R}$ & $0,0 \pm 0,5 \mathrm{a}$ & $\mathrm{R}$ & $0,0 \mathrm{a}$ \\
\hline SCSA 64 & T. bicolor & $0,0 \pm 0,5 \mathrm{a}$ & $0,0 \pm 0,4 \mathrm{a}$ & $0,0 \pm 0,4 \mathrm{a}$ & $0,0 \mathrm{a}$ & $0,0 \pm 0,4 \mathrm{a}$ & $\mathrm{R}$ & $0,0 \pm 0,4 \mathrm{a}$ & $\mathrm{R}$ & $0,0 \pm 0,4 \mathrm{a}$ & $\mathrm{R}$ & $0,0 \mathrm{a}$ \\
\hline SCSA 177 & T. cacao & $0,0 \pm 0,5 \mathrm{a}$ & $0,0 \pm 0,5 \mathrm{a}$ & $0,0 \pm 0,5 \mathrm{a}$ & $0,0 \mathrm{a}$ & $0,0 \pm 0,5 \mathrm{a}$ & $\mathrm{R}$ & $0,0 \pm 0,5 \mathrm{a}$ & $\mathrm{R}$ & $0,0 \pm 0,5 \mathrm{a}$ & $\mathrm{R}$ & $0,0 \mathrm{a}$ \\
\hline SCSA 41 & T. bicolor & $0,0 \pm 0,5 \mathrm{a}$ & $0,0 \pm 0,5 \mathrm{a}$ & $0,0 \pm 0,5 \mathrm{a}$ & $0,0 \mathrm{a}$ & $0,0 \pm 0,5 \mathrm{a}$ & $\mathrm{R}$ & $0,0 \pm 0,5 \mathrm{a}$ & $\mathrm{R}$ & $0,0 \pm 0,5 \mathrm{a}$ & $\mathrm{R}$ & $0,0 \mathrm{a}$ \\
\hline SCSA 103 & T. cacao & $0,3 \pm 0,5 \mathrm{ab}$ & $0,3 \pm 0,4 \mathrm{ab}$ & $1,8 \pm 0,4 \mathrm{a}-\mathrm{g}$ & $0,8 \mathrm{a}-\mathrm{c}$ & $0,0 \pm 0,5 \mathrm{a}$ & $\mathrm{R}$ & $1,0 \pm 0,4 a-d$ & $\mathrm{R}$ & $1,7 \pm 0,4 \mathrm{a}-\mathrm{g}$ & MR & $0,9 \mathrm{a}-\mathrm{e}$ \\
\hline SCSA 27 & T. cacao & $0,3 \pm 0,4 \mathrm{ab}$ & $0,7 \pm 0,4 a-d$ & $2,0 \pm 0,5 \mathrm{a}-\mathrm{h}$ & $1,0 a-d$ & $0,3 \pm 0,4 \mathrm{a}-\mathrm{c}$ & $\mathrm{R}$ & $0,3 \pm 0,4 a-c$ & $\mathrm{R}$ & $1,0 \pm 0,5 \mathrm{a}-\mathrm{d}$ & $\mathrm{R}$ & $0,6 a-c$ \\
\hline SCSA 29 & T. cacao & $1,0 \pm 0,5 \mathrm{a}-\mathrm{e}$ & $3,0 \pm 0,5 \mathrm{a}-\mathrm{h}$ & $2,0 \pm 0,5 \mathrm{a}-\mathrm{h}$ & $2,0 \mathrm{~b}-\mathrm{g}$ & $1,0 \pm 0,5 \mathrm{a}-\mathrm{d}$ & $\mathrm{R}$ & $3,0 \pm 0,5 b-i$ & MS & $2,0 \pm 0,5 \mathrm{a}-\mathrm{h}$ & MR & $2,0 \mathrm{c}-\mathrm{i}$ \\
\hline SCSA 71 & T. cacao & $1,7 \pm 0,4 \mathrm{a}-\mathrm{g}$ & $0,0 \pm 0,5 \mathrm{a}$ & $5,0 \pm 0,5 \mathrm{~h}$ & $2,2 \mathrm{c}-\mathrm{h}$ & $1,5 \pm 0,4 a-f$ & MR & $0,0 \pm 0,5 \mathrm{a}$ & $\mathrm{R}$ & $5,0 \pm 0,5 \mathrm{i}$ & S & $2,2 d-j$ \\
\hline SCSA 90 & T. cacao & $1,8 \pm 0,5 \mathrm{a}-\mathrm{g}$ & $0,3 \pm 0,5 \mathrm{ab}$ & $0,3 \pm 0,5 \mathrm{ab}$ & $0,8 \mathrm{a}-\mathrm{c}$ & $2,3 \pm 0,5 \mathrm{a}-\mathrm{i}$ & MR & $1,0 \pm 0,5 \mathrm{a}-\mathrm{d}$ & $\mathrm{R}$ & $0,3 \pm 0,5 \mathrm{ab}$ & $\mathrm{R}$ & $1,2 \mathrm{a}-\mathrm{f}$ \\
\hline SCSA 88 & T. cacao & $1,8 \pm 0,4 \mathrm{a}-\mathrm{g}$ & $2,8 \pm 0,4 \mathrm{a}-\mathrm{h}$ & $2,6 \pm 0,4 a-h$ & $2,4 c-i$ & $2,3 \pm 0,4 \mathrm{a}-\mathrm{i}$ & MR & $3,9 \pm 0,4 \mathrm{~d}-\mathrm{i}$ & S & $3,7 \pm 0,4 \mathrm{~d}-\mathrm{i}$ & MS & 3,3i-m \\
\hline SCSA 175 & T. cacao & $1,9 \pm 0,4 \mathrm{a}-\mathrm{h}$ & $1,7 \pm 0,4 \mathrm{a}-\mathrm{g}$ & $1,4 \pm 0,4 a-f$ & $1,7 \mathrm{a}-\mathrm{f}$ & $2,0 \pm 0,4 \mathrm{a}-\mathrm{h}$ & MR & $2,1 \pm 0,4 a-i$ & MR & $1,3 \pm 0,4 \mathrm{a}-\mathrm{e}$ & MR & $1,8 b-i$ \\
\hline SCSA 174 & T. cacao & $2,0 \pm 0,5 \mathrm{a}-\mathrm{h}$ & $1,0 \pm 0,5 \mathrm{a}-\mathrm{e}$ & $1,5 \pm 0,5 \mathrm{a}-\mathrm{f}$ & $1,5 \mathrm{a}-\mathrm{e}$ & $1,0 \pm 0,5 \mathrm{a}-\mathrm{d}$ & $\mathrm{R}$ & $0,5 \pm 0,5 \mathrm{a}-\mathrm{c}$ & $\mathrm{R}$ & $1,0 \pm 0,5 \mathrm{a}-\mathrm{d}$ & $\mathrm{R}$ & $0,8 \mathrm{a}-\mathrm{e}$ \\
\hline SCSA 143 & T. bicolor & $2,0 \pm 0,5 \mathrm{a}-\mathrm{h}$ & $0,0 \pm 0,5 \mathrm{a}$ & $3,0 \pm 0,5 \mathrm{a}-\mathrm{h}$ & $1,7 \mathrm{a}-\mathrm{f}$ & $1,0 \pm 0,5 \mathrm{a}-\mathrm{d}$ & $\mathrm{R}$ & $0,00 \pm 0,5 \mathrm{a}$ & $\mathrm{R}$ & $3,0 \pm 0,5 b-i$ & MS & $1,3 a-g$ \\
\hline SCSA 151 & T. cacao & $2,0 \pm 0,5 \mathrm{a}-\mathrm{h}$ & $0,5 \pm 0,5 \mathrm{a}-\mathrm{c}$ & $0,0 \pm 0,5 \mathrm{a}$ & $0,8 \mathrm{a}-\mathrm{d}$ & $2,0 \pm 0,5 \mathrm{a}-\mathrm{h}$ & MR & $0,5 \pm 0,5 \mathrm{a}-\mathrm{c}$ & $\mathrm{R}$ & $0,5 \pm 0,5 \mathrm{a}-\mathrm{c}$ & $\mathrm{R}$ & $1,0 \mathrm{a}-\mathrm{f}$ \\
\hline SCSA 3 & T. cacao & $2,5 \pm 0,5 \mathrm{a}-\mathrm{h}$ & $3,3 \pm 0,4 b-h$ & $1,7 \pm 0,4 \mathrm{a}-\mathrm{g}$ & $2,5 \mathrm{~d}-\mathrm{i}$ & $2,5 \pm 0,5 \mathrm{a}-\mathrm{i}$ & MR & $5,0 \pm 0,4 \mathrm{i}$ & S & $1,7 \pm 0,4 \mathrm{a}-\mathrm{g}$ & MR & $3,1 \mathrm{~h}-1$ \\
\hline SCSA 140 & T. cacao & $2,5 \pm 0,5 \mathrm{a}-\mathrm{h}$ & $0,0 \pm 0,5 \mathrm{a}$ & $5,0 \pm 0,5 \mathrm{~h}$ & $2,5 \mathrm{~d}-\mathrm{i}$ & $2,5 \pm 0,5 \mathrm{a}-\mathrm{i}$ & MR & $1,0 \pm 0,5 \mathrm{a}-\mathrm{d}$ & $\mathrm{R}$ & $5,0 \pm 0,5 \mathrm{i}$ & $\mathrm{S}$ & $2,8 \mathrm{~g}-\mathrm{k}$ \\
\hline SCSA115 & T. grandiflorum & $3,0 \pm 0,5 \mathrm{a}-\mathrm{h}$ & $2,0 \pm 0,5 \mathrm{a}-\mathrm{h}$ & $2,0 \pm 0,5 a-h$ & $2,3 c-h$ & $2,0 \pm 0,5 \mathrm{a}-\mathrm{h}$ & MR & $2,0 \pm 0,5 \mathrm{a}-\mathrm{h}$ & MR & $2,0 \pm 0,5 \mathrm{a}-\mathrm{h}$ & MR & $2,0 \mathrm{c}-\mathrm{i}$ \\
\hline SCSA 75 & T. cacao & $3,0 \pm 0,5 \mathrm{a}-\mathrm{h}$ & $3,0 \pm 0,5 \mathrm{a}-\mathrm{h}$ & $3,0 \pm 0,5 \mathrm{a}-\mathrm{h}$ & $3,0 \mathrm{~d}-\mathrm{j}$ & $2,0 \pm 0,5 \mathrm{a}-\mathrm{h}$ & MR & $3,0 \pm 0,5 b-i$ & MS & $2,5 \pm 0,5 \mathrm{a}-\mathrm{i}$ & MR & $2,5 \mathrm{f}-\mathrm{j}$ \\
\hline SCSA 152 & T. grandiflorum & $3,0 \pm 0,5 \mathrm{a}-\mathrm{h}$ & $4,0 \pm 0,5 \mathrm{e}-\mathrm{h}$ & $0,0 \pm 0,5 \mathrm{a}$ & $2,3 c-h$ & $3,0 \pm 0,5 \mathrm{~b}-\mathrm{i}$ & MS & $4,0 \pm 0,5 \mathrm{e}-\mathrm{i}$ & $\mathrm{S}$ & $0,0 \pm 0,5 \mathrm{a}$ & $\mathrm{R}$ & $2,3 e-j$ \\
\hline SCSA 121 & T. grandiflorum & $3,0 \pm 0,5 \mathrm{a}-\mathrm{h}$ & $2,0 \pm 0,5 \mathrm{a}-\mathrm{h}$ & $2,0 \pm 0,5 \mathrm{a}-\mathrm{h}$ & $2,3 c-h$ & $3,5 \pm 0,5 \mathrm{~d}-\mathrm{i}$ & MS & $2,5 \pm 0,5 \mathrm{a}-\mathrm{i}$ & MR & $2,5 \pm 0,5 \mathrm{a}-\mathrm{i}$ & MR & $2,8 \mathrm{~g}-\mathrm{k}$ \\
\hline SCSA 32 & T. bicolor & $3,0 \pm 0,5 \mathrm{a}-\mathrm{h}$ & $4,0 \pm 0,5 \mathrm{e}-\mathrm{h}$ & $3,5 \pm 0,5 \mathrm{c}-\mathrm{h}$ & $3,5 \mathrm{~g}-\mathrm{k}$ & $2,0 \pm 0,5 \mathrm{a}-\mathrm{h}$ & MR & $3,0 \pm 0,5 b-i$ & MS & $2,5 \pm 0,5 \mathrm{a}-\mathrm{i}$ & MR & $2,5 \mathrm{f}-\mathrm{j}$ \\
\hline SCSA 28 & T. cacao & $3,0 \pm 0,5 \mathrm{a}-\mathrm{h}$ & $0,0 \pm 0,5 \mathrm{a}$ & $0,0 \pm 0,5 \mathrm{a}$ & $1,0 a-d$ & $2,0 \pm 0,5 \mathrm{a}-\mathrm{h}$ & MR & $0,0 \pm 0,5 \mathrm{a}$ & $\mathrm{R}$ & $0,0 \pm 0,5 \mathrm{a}$ & $\mathrm{R}$ & $0,7 \mathrm{a}-\mathrm{d}$ \\
\hline SCSA 18 & T. cacao & $3,0 \pm 0,5 \mathrm{a}-\mathrm{h}$ & $2,0 \pm 0,5 \mathrm{a}-\mathrm{h}$ & $1,0 \pm 0,5 \mathrm{a}-\mathrm{e}$ & $2,0 \mathrm{~b}-\mathrm{g}$ & $3,0 \pm 0,5 \mathrm{~b}-\mathrm{i}$ & MS & $1,0 \pm 0,5 \mathrm{a}-\mathrm{d}$ & $\mathrm{R}$ & $1,0 \pm 0,5 \mathrm{a}-\mathrm{d}$ & $\mathrm{R}$ & $1,7 \mathrm{~b}-\mathrm{h}$ \\
\hline SCSA 6 & T. cacao & $3,3 \pm 0,5 b-h$ & $3,3 \pm 0,5 b-h$ & $3,0 \pm 0,5 \mathrm{a}-\mathrm{h}$ & $3,2 e-j$ & $3,3 \pm 0,5 \mathrm{c}-\mathrm{i}$ & MS & $3,8 \pm 0,5 \mathrm{~d}-\mathrm{i}$ & S & $4,0 \pm 0,5 \mathrm{e}-\mathrm{i}$ & S & $3,7 \mathrm{j}-\mathrm{n}$ \\
\hline SCSA 160 & T. grandiflorum & $3,5 \pm 0,5 \mathrm{c}-\mathrm{h}$ & $3,0 \pm 0,5 \mathrm{a}-\mathrm{h}$ & $4,0 \pm 0,4 \mathrm{e}-\mathrm{h}$ & $3,5 \mathrm{~g}-\mathrm{k}$ & $2,5 \pm 0,5 \mathrm{a}-\mathrm{i}$ & MR & $2,0 \pm 0,5 \mathrm{a}-\mathrm{h}$ & MR & $3,0 \pm 0,5 b-i$ & MS & $2,5 \mathrm{f}-\mathrm{j}$ \\
\hline SCSA 45 & T.cacao & $3,5 \pm 0,5 \mathrm{c}-\mathrm{h}$ & $4,3 \pm 0,5 \mathrm{f}-\mathrm{h}$ & $3,8 \pm 0,5 \mathrm{~d}-\mathrm{h}$ & $3,8 \mathrm{~h}-\mathrm{k}$ & $3,8 \pm 0,5 \mathrm{~d}-\mathrm{i}$ & MS & $4,0 \pm 0,5 \mathrm{e}-\mathrm{i}$ & $\mathrm{S}$ & $3,0 \pm 0,5 b-i$ & MS & $3,6 \mathrm{j}-\mathrm{n}$ \\
\hline SCSA 108 & T. cacao & $3,7 \pm 0,4 \mathrm{~d}-\mathrm{h}$ & $4,2 \pm 0,4 \mathrm{f}-\mathrm{h}$ & $5,0 \pm 0,5 \mathrm{~h}$ & $4,3 \mathrm{jk}$ & $4,7 \pm 0,4 \mathrm{~h}-\mathrm{i}$ & $\mathrm{S}$ & $4,2 \pm 0,4 \mathrm{e}-\mathrm{i}$ & $\mathrm{S}$ & $5,0 \pm 0,5 \mathrm{i}$ & $\mathrm{S}$ & $4,61-n$ \\
\hline SCSA 50 & T. cacao & $3,7 \pm 0,5 \mathrm{~d}-\mathrm{h}$ & $3,2 \pm 0,5 b-h$ & $4,3 \pm 0,4 \mathrm{f}-\mathrm{h}$ & $3,7 \mathrm{hk}$ & $4,2 \pm 0,5 \mathrm{e}-\mathrm{i}$ & S & $4,3 \pm 0,5 \mathrm{f}-\mathrm{i}$ & $\mathrm{S}$ & $4,4 \pm 0,4 \mathrm{~g}-\mathrm{i}$ & S & $4,3 \mathrm{k}-\mathrm{n}$ \\
\hline SCSA 169 & T. grandiflorum & $4,0 \pm 0,5 \mathrm{e}-\mathrm{h}$ & $3,0 \pm 0,5 \mathrm{a}-\mathrm{h}$ & $3,5 \pm 0,5 \mathrm{c}-\mathrm{h}$ & $3,5 \mathrm{~g}-\mathrm{k}$ & $3,0 \pm 0,5 b-i$ & MS & $2,0 \pm 0,5 a-h$ & MR & $2,5 \pm 0,5 \mathrm{a}-\mathrm{i}$ & MR & $2,5 \mathrm{f}-\mathrm{j}$ \\
\hline SCSA 73 & T. grandiflorum & $4,0 \pm 0,5 \mathrm{e}-\mathrm{h}$ & $5,0 \pm 0,5 \mathrm{~h}$ & $5,0 \pm 0,5 \mathrm{~h}$ & $4,7 \mathrm{jk}$ & $4,0 \pm 0,5 \mathrm{e}-\mathrm{i}$ & $\mathrm{S}$ & $5,0 \pm 0,5 \mathrm{i}$ & $\mathrm{S}$ & $5,0 \pm 0,5 \mathrm{i}$ & $\mathrm{S}$ & $4,7 \mathrm{mn}$ \\
\hline SCSA 42 & T. cacao & $4,5 \pm 0,5 \mathrm{f}-\mathrm{h}$ & $4,0 \pm 0,5 \mathrm{e}-\mathrm{h}$ & $4,8 \pm 0,5 \mathrm{~g}-\mathrm{h}$ & $4,4 \mathrm{jk}$ & $4,3 \pm 0,5 \mathrm{e}-\mathrm{i}$ & S & $4,3 \pm 0,5 \mathrm{e}-\mathrm{i}$ & S & $4,5 \pm 0,5 \mathrm{~g}-\mathrm{i}$ & S & $4,3 \mathrm{k}-\mathrm{n}$ \\
\hline SCSA 43 & T. cacao & $5,0 \pm 0,5 \mathrm{~h}$ & $3,0 \pm 0,5 \mathrm{a}-\mathrm{h}$ & $5,0 \pm 0,5 \mathrm{~h}$ & $4,3 \mathrm{jk}$ & $5,0 \pm 0,5 \mathrm{i}$ & S & $1,0 \pm 0,5 \mathrm{a}-\mathrm{d}$ & $\mathrm{R}$ & $5,0 \pm 0,5 \mathrm{i}$ & S & $3,7 \mathrm{j}-\mathrm{n}$ \\
\hline SCSA 5 & T. cacao & $5,0 \pm 0,5 \mathrm{~h}$ & $5,0 \pm 0,5 \mathrm{~h}$ & $5,0 \pm 0,5 \mathrm{~h}$ & $5,0 \mathrm{k}$ & $5,0 \pm 0,5 \mathrm{i}$ & S & $5,0 \pm 0,5 \mathrm{i}$ & S & $5,0 \pm 0,5 \mathrm{i}$ & S & $5,0 \mathrm{n}$ \\
\hline SCSA 180 & T. cacao & $5,0 \pm 0,5 \mathrm{~h}$ & $5,0 \pm 0,5 \mathrm{~h}$ & $5,0 \pm 0,5 \mathrm{~h}$ & $5,0 \mathrm{k}$ & $5,0 \pm 0,5 \mathrm{i}$ & S & $5,0 \pm 0,5 i$ & S & $5,0 \pm 0,5 \mathrm{i}$ & S & $5,0 \mathrm{n}$ \\
\hline SCSA 178 & T. cacao & $5,0 \pm 0,5 \mathrm{~h}$ & $0,0 \pm 0,5 \mathrm{a}$ & $5,0 \pm 0,5 \mathrm{~h}$ & $3,3 \mathrm{f}-\mathrm{k}$ & $4,0 \pm 0,5 \mathrm{e}-\mathrm{i}$ & S & $0,0 \pm 0,5 \mathrm{a}$ & $\mathrm{R}$ & $5,0 \pm 0,5 \mathrm{i}$ & S & $3,0 \mathrm{~h}-\mathrm{k}$ \\
\hline SCSA 107 & T. cacao & $5,0 \pm 0,5 \mathrm{~h}$ & $5,0 \pm 0,5 \mathrm{~h}$ & $5,0 \pm 0,5 \mathrm{~h}$ & $5,0 \mathrm{k}$ & $5,0 \pm 0,5 \mathrm{i}$ & S & $5,0 \pm 0,5 \mathrm{i}$ & S & $5,0 \pm 0,5 \mathrm{i}$ & $\mathrm{S}$ & $5,0 \mathrm{n}$ \\
\hline SCSA 179 & T. cacao & $5,0 \pm 0,5 \mathrm{~h}$ & $0,0 \pm 0,5 \mathrm{a}$ & $1,0 \pm 0,5 \mathrm{a}-\mathrm{e}$ & $2,0 \mathrm{~b}-\mathrm{g}$ & $5,0 \pm 0,5 \mathrm{i}$ & S & $0,0 \pm 0,5 \mathrm{a}$ & $\mathrm{R}$ & $1,0 \pm 0,5 \mathrm{a}-\mathrm{d}$ & $\mathrm{R}$ & $2,0 \mathrm{c}-\mathrm{i}$ \\
\hline SCSA 16 & T. cacao & $5,0 \pm 0,5 \mathrm{~h}$ & $4,2 \pm 0,5 \mathrm{f}-\mathrm{h}$ & $3,0 \pm 0,4 a-h$ & $4,1 \mathrm{i}-\mathrm{k}$ & $5,0 \pm 0,5 \mathrm{i}$ & S & $4,7 \pm 0,5 \mathrm{hi}$ & $\mathrm{S}$ & $3,1 \pm 0,4 \mathrm{~b}-\mathrm{i}$ & MS & $4,3 \mathrm{k}-\mathrm{n}$ \\
\hline Media $^{\mathrm{d}}$ & & $2,1 b$ & $1,8 \mathrm{a}$ & $2,1 \mathrm{ab}$ & 2.0 & $2,0 \mathrm{a}$ & & $1,9 \mathrm{a}$ & & $2,0 \mathrm{a}$ & & 1,96 \\
\hline
\end{tabular}

${ }^{a}$ Tipo de Reacción (TR): R: Resistente (0 - 1,25); MR: Moderadamente Resistente (1,26 - 2,50); MS: Moderadamente susceptible (2,51 - 3,75); S: Susceptible (3,76 - 5); ${ }^{\mathrm{b}}$ Error estándar; ${ }^{\mathrm{c}}$ Valores en cada columna seguidos por la misma letra no difieren estadísticamente (Prueba Tukey HSD; P $<0,05$ ); ${ }^{\mathrm{d}}$ Medias de los valores en esta fila seguidas por la misma letra no difieren estadísticamente (Prueba Tukey HSD; P <0,05)

Tabla 3 Medias de severidad externa (SE) e interna (SI) de moniliasis producida por inoculación controlada de M. roreri en 50 materiales genéticos de Theobroma (T. cacao, T. grandiflorum y T. bicolor) seleccionados en Caquetá (Colombia). 


\section{REFERENCIAS BIBLIOGRÁFICAS}

1. Aránzazu, H.F.; Martínez N.; Palencia, G.; Coronado, R.; Rincón, D. Cacao: Mejoramiento genético para incrementar la producción del sistema de cacao en Colombia. Unión temporal cacao de Colombia Uno: Federación Nacional de Cacaoteros, Ministerio de Agricultura y Desarrollo Rural y Corpoica, 2009.

2. Arciniegas - Leal, A. Caracterización de árboles superiores de cacao (Theobroma cacao L.) seleccionados por el programa de mejoramiento genético del CATIE. Tesis (Magíster Scientiae) - Programa de Enseñanza para el Desarrollo y la Conservación - Instituto Agronómico Tropical de Investigación y Enseñanza, Turrialba, Costa Rica. 144p. 2005.

3. Castellanos, O. L.; Torres, S.; Fonseca, V.; Montanez, A.; Sánchez. AgenA da prospectiva de investigación y desarrollo tecnológico para la cadena productiva de cacao-chocolate en Colombia. Grupo de Investigación y Desarrollo en Gestión, Productividad y Competitividad - Biogestión, Universidad Nacional de Colombia; Ministerio de Agricultura y Desarrollo Rural - MADR, Bogotá. 2007.

4. Centro Agronómico Tropical de Investigaciones y Enseñanza, CATIE. Inforł me de progreso anual. Serie institucional. 8ed, Catie, Turrialba, p. 160, 1987.

5. Di Rienzo, J.A.; Casanoves, F.; Balzarini, M. G.; Gonzalez, L.; Tablada, M.; Robledo, C.W. Grupo InfoStat, FCA, Universidad Nacional de Córdoba, Argentina. InfoStat versión 2013. URL http://www.infostat.com.ar

6. Evans, H.C. Pod rot of cacao caused by Moniliophthora (Monilia) roreri. Phytopathological Papers, p. 24 - 44, 1981.

7. Evans, H. Cacao diseases - the trilogy revisited. Phytopathology, v. 97, p. 1640-1643, 2007.

8. Federación Nacional de Cacaoteros de Colombia, FEDECACAO. Caracterización físico - química y beneficio del grano de cacao (Theobroma cacao) en Colombia, Bogotá D.C. Produmedios, 31 p. 2005.

9. Hernández, M., Barrera, J. "Frutas amazónicas Competitividad e innovación”. Editorial Scripto Ltda. v. 1, 100p. 2009.

10. Instituto de Hidrología, Meteorología y Estudios Ambientales, IDEAM. Datos meteorológicos del Departamento del Caquetá. Estación Limnimétrica de Montañita (Caquetá), Bogotá, p. 3. 2009

11. Instituto Geográfico Agustín Codazzi, IGAC. Caquetá, características geográficas. Imprenta Nacional de Colombia, Bogotá, p. 373, 2010.

12. Jaimes, Y.; Aranzazu, F.; Rodriguez, E.; Martinez, N. Behavior of introduced regional clones of Theobroma cacao toward the infection of Moniliophthora roreri in three different regions of Colombia. Agronomía Colombiana, Santander, v. 29 n.1, p. 171-178, 2011.

13. Krauss, U.; Hoopen, G.; Hidalgo, E.; Martinez, A.; Stirrup, T.; Arroyo, C.; Garcia, J.; Palacios, M. The effect of cane molasses amendment on biocontrol of frosty pod rot (Moniliophthora roreri) and black pod (Phythothora spp.) of cocoa (Theobroma cacao) in Panama. Biological control, v. 39, p. 232-239, 2006.

14. Melgar, J.Y.; González, A. Resultados de evaluación de la reacción de germoplasma de cacao a la moniliasis en Honduras por medio de inoculación artificial. Fundación Hondureña de Investigación Agrícola (FHIA). Departamento de Protección vegetal. Programa de cacao y Agroforestería. Informe Técnico, p. 45-50, 2011.
15. Melgar, J.; Reyes, Z.; Rivera, J.; Sánchez, J.; Dubón, A. Avances en la evaluación en Honduras de germoplasma de cacao presumiblemente resistente a la moniliasis. Fundación Hondureña de Investigación Agrícola (FHIA). Programa de cacao y Agroforestería. Departamento de protección vegetal Informe Técnico, p. 6, 2012.

16. Munar, F. Quintero, L. Sterling, A. 2014. Caracterización molecular de materiales del género Theobroma (T. cacao, T. grandiflorum y T. bicolor) seleccionados en Caquetá (Colombia). In: Sterling, A., Rodríguez, C. Selección de materiales sobresalientes del género Theobroma como estrategia de rescate del germoplasma local en el departamento del Caquetá. Bogotá-Colombia: Instituto Amazónico de Investigaciones Científicas Sinchi, 2014. V. 1, cap. 4, p. 91-105.

17. Phillips-Mora, W. Studies at CATIE on moniliasis resistance (Moniliophthora roreri) International Workshop on the Contribution of Disease Resistance to Cocoa Variety Improvement, 1999. Bahía, p. 111-117, 1996.

18. Phillips-Mora W.; Krauss, U.; Evans, H.; Wilkinson, M. Genetic diversity of the cacao pathogen Moniliophthora roreri (Cif.) Evans et al. in tropical America. Proceedings of the 14th International Cocoa Research Conference, Accra, Ghama cocoa producers Alliance, (Abstract 60). 2003.

19. Phillips-Mora, W.; Castillo, J.; Krauss, U.; Rodríguez, E.; Wilkinson, M. Evaluation of cacao (Theobroma cacao) clones against seven Colombian isolates of Moniliophthora roreri from four pathogen genetic groups. Plant Pathology, Oxford, v. 54, p. 483-490, 2005

20. Phillips-Mora, W.; Coutiño, A.; Ortiz, C. F.; López, A. P., Hernández, J.; Aime, M. C. First report of Moniliophthora roreri causing frosty pod rot (= moniliasis disease) of cacao in Mexico. Plant Pathology. v. 55, p. 584, 2006.

21. Phillips-Mora, W.; Arciniegas-Leal, A.; Mata-Quirós, A.; MotamayorArias, J. C. Catálogo de clones de cacao seleccionados por el CATIE para siembras comerciales. Centro Agronómico Tropical de Investigación y Enseñanza (CATIE). Programa de Mejoramiento Genético de Cacao, Turrialba, p. 68, 2012

22. Polanco, L.; Medina, J. Caracterización de clones de cacao por la respuesta a Monilia (Moniliophthora roreri (Cif \& Par) Evans et al.,) en Santander. Fitopatología Colombiana. Palmira. v. 28, n. 2, p. 61-64, 2005.

23. Porras, V. Determinación de la estabilidad de la resistencia a Monilia roreri en cultivares de cacao en dos zonas de Costa Rica. Tesis (Magister Scientie) - Facultad de Protección Vegetal- Centro Agronómico Tropical de Investigación y Enseñanza, Turrialba. 124p, 1985.

24. Ribeiro, F.; Parlevleliet, J.; Zambolim, L. Concepts in plant disease resisf tance. Fitopatologia, Brasilera, v. 26, p. 577-589, 2001

25. Santos, C.R.; Pires, J.L.; Correa, R.X. Morphological characterization of leaf, flower, fruit and seed traits among Brazilian Theobroma L. species. Genet Resour Crop Evol (2012). Centro de Pesquisas do Cacau (CEPEC-CEPLAC), Ilhéus, v. 59, p. 327-345, 2012.

26. Sánchez, J.A.; Brenes, O.; Phillips, W.; Enríquez, G. Metodología para la inoculación de mazorcas de cacao con el hongo Moniliophthora rorer (Monilia). Proceedings of the 10th International Cocoa Research Confere ence (1988), Santo Domingo, p.467-472, 1987.

27. Suárez, C.; Amores, F.; Lopez, O. New sources to resistance to Moniliophthora roreri. Developing Effective Sustainable Crop Protection Systems for Increased Cocoa Production. National Cocoa Program, INIAP. 2006 